Trauma Berufskrankh 2009 · 11[Suppl 1]:97-106 DOI 10.1007/s10039-008-1443-4

Online publiziert: 3. Oktober 2008

(c) Springer Medizin Verlag 2008

\section{T. Mittlmeier · M. Beck}

Abteilung für Unfall- und Wiederherstellungschirurgie,

Chirurgische Klinik und Poliklinik, Universität Rostock

\title{
Verletzungen von Becken und Azetabulum
}

\section{Fehler, Fallstricke und Lösungswege}

\section{Problem}

Frakturen von Becken und Azetabulum machen lediglich $2 \%$ aller Brüche aus bzw. treten mit einer niedrigen Inzidenz von 37/100.00o Einwohner/Jahr auf $[6,10,15]$. Dementsprechend sind die individuellen Erfahrungen mit diesen Verletzungen auch an größeren Traumazentren begrenzt, und es liegen, trotz mehrerer erfolgreicher multizentrischer Studien unter dem Dach der DGU und der Deutschen Sektion der AO International [13, $14,15]$ nur wenige verbindliche Erkenntnisse vor, die den strengen Kriterien der evidenzbasierten Medizin genügen. Expertentreffen mit entsprechenden Versuchen einer Konsensusbildung zu strittigen Fragen des diagnostischen und therapeutischen Prozedere spiegeln derzeit den aktuellen Wissensstand zum Thema besser wider $[13,15]$. Die Konzentration von Patienten mit den im Regelfall prognostisch ernsten Verletzungstypen auf große Zen-

\begin{tabular}{|ll|}
\hline \multicolumn{2}{|l|}{ Abkürzungen } \\
\hline$A G \quad$ & Arbeitsgemeinschaft \\
\hline$A O$ & $\begin{array}{l}\text { Arbeitsgemeinschaft für } \\
\text { Osteosynthesefragen }\end{array}$ \\
\hline$B W K$ & Brustwirbelkörper \\
\hline$C T$ & Computertomographie \\
\hline$D G U$ & $\begin{array}{l}\text { Deutsche Gesellschaft für } \\
\text { Unfallchirurgie }\end{array}$ \\
\hline FKN & Femurkopfnekrose \\
\hline$H O$ & Heterogene Ossifikation \\
\hline LWK & Lendenwirbelkörper \\
\hline ORIF & "open reduction, internal fixation“ \\
\hline$T E P$ & Totalendoprothese \\
\hline
\end{tabular}

tren mit entsprechender Erfahrung ist eine logische Konsequenz.

Seit dem systematischen Beginn einer Klassifikation in einfache und kombinierte Frakturformen bei der Azetabulum- $[4,6]$ und der Beckenringfraktur nach dem Grad der Instabilität und den Kriterien der AO [7] als Grundlage einer differenzierten Therapiewahl, dem Erarbeiten von Standards bei der Diagnostik und der Zugangswahl im Rahmen der operativen Therapie sind technische Neuerungen wie die heute praktisch ubiquitär verfügbare Computertomographie hinzugekommen und zahlreiche Optimierungen im Management dieser Verletzungen und Modifikationen, insbesondere der operativen Therapieoptionen, vorgeschlagen worden $[1,3,11,12,19,21,22$, 24].

Betrachtet man hinsichtlich des Outcome lediglich die Mortalität, ist heute bekannt, dass nicht der Verletzungstyp und die Art der Beckeninstabilität entscheidend sind, sondern die Begleitverletzungen [8], etwa das begleitende Weichteiltrauma beim Komplextrauma des Be- ckens $[14,15]$. Eine anatomiegerechte Rekonstruktion der jeweiligen Verletzung ist zwar keine Garantie, aber offenbar eine wichtige Voraussetzung für ein gutes $\mathrm{Re}$ sultat $[2,10,22]$. Bei der Analyse der funktionellen Resultate - ermittelt mit unterschiedlichen gemischten Scores - erreicht die Zahl guter und exzellenter Resultate sowohl bei Beckenring- als auch bei Azetabulumfrakturen zwischen 75 und $85 \%$ $[2,5,22]$. Dies darf jedoch nicht darüber hinweg täuschen, dass je nach Verletzungstyp erhebliche Unterschiede bestehen können und eine spezifische Evaluation der Lebensqualität nach Beckenringund Azetabulumfrakturen ein deutlich schlechteres Ergebnis als anhand eines üblichen klinischen Scores ermittelt ergibt [23]. Zudem betragen die kumulativen perioperativen Komplikationsraten bei beiden Verletzungsentitäten über 50\% $[9,18]$, und unerwünschte Langzeitfolgen sind ebenfalls beachtlich hoch $([6,7,16]$, - Tab. 1).

Ein wesentliches Element der Behandlungsstrategie kann also nur sein, an die individuelle Situation adaptiert frühzei-

\begin{tabular}{|lll}
\hline Tab. 1 & Langzeitfolgen nach Azetabulum- und Beckenringfraktur. (Nach [7]) \\
\hline & Azetabulumfraktur [\%] & Beckenringfraktur [\%] \\
Arthrose & 35 & 9 \\
\hline Beckenschiefstand & 16 & 38 \\
\hline FKN & 6 & \\
HO & 14 & $<1$ \\
\hline Pseudarthrose & $<1$ & 11 \\
\hline Neurologische Ausfälle & 14 & 6 \\
\hline Sexuelle Dysfunktion & & 2 \\
\hline Urologische Probleme & &
\end{tabular}



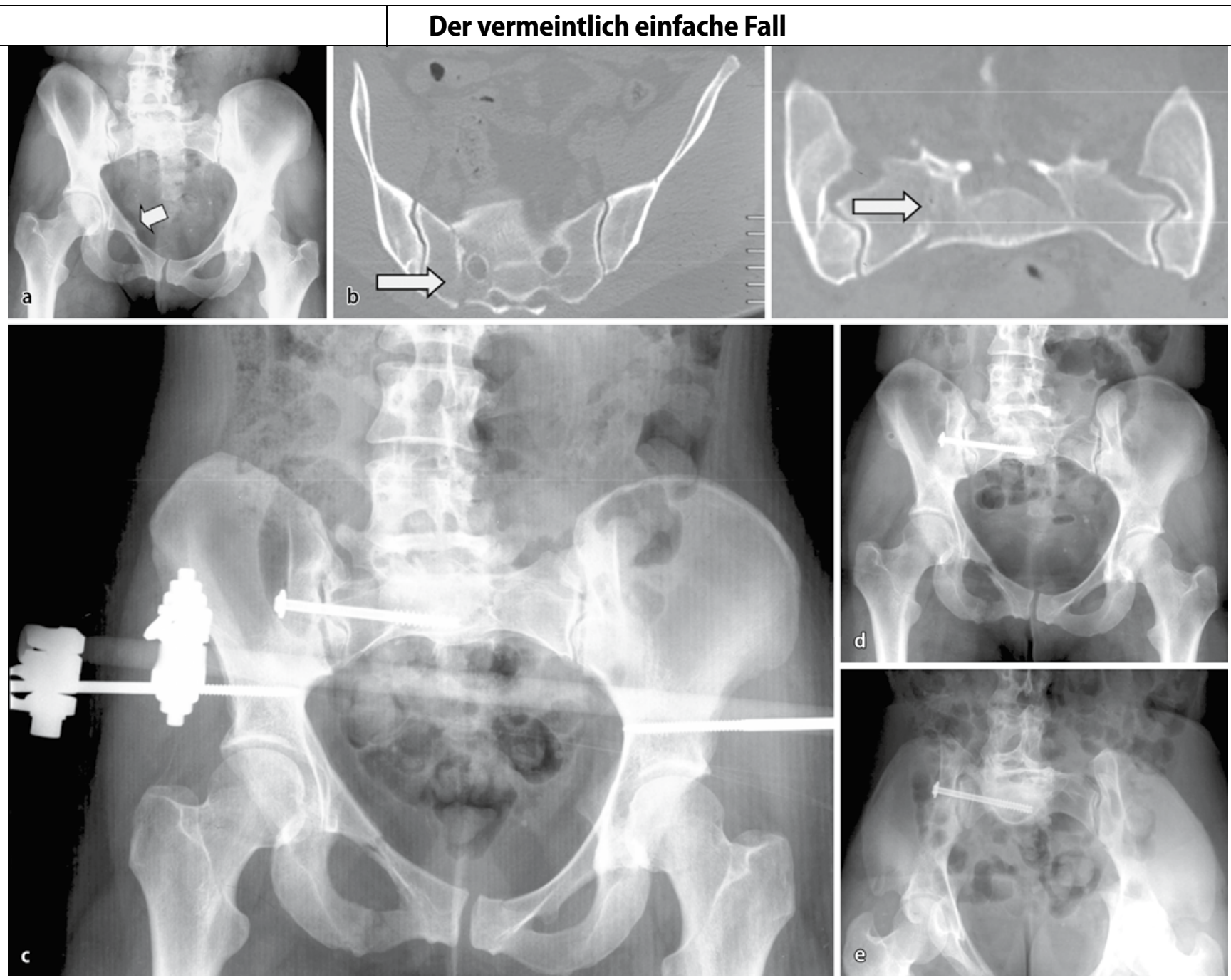

Abb. 1 \ Fall 1, 32-jährige Patientin, AO-Fraktur 61B2.1., a a.-p. Beckenübersichtsaufnahme, b CT: Kompressionsfraktur der rechtsseitigen Massa lateralis, c a.-p. Beckenübersichtsaufnahme postoperativ, d,e Beckenübersichts- und Inlet-Aufnahme nach Entfernung des Fixateurs und Vollmobilisation, weitere Erläuterungen s. Kasuistik

tig einen geeigneten Behandlungspfad mit minimierten Risikoaussichten einzuschlagen. Im Folgenden sollen deshalb eine Auswahl typischer Fehlerkonstellationen der frühen posttraumatischen Phase aufgezeigt werden sowie die jeweiligen Lösungsansätze bzw. eine entsprechende Fehlervermeidungsstrategie. Aufgrund der Vielfalt des Themas ist diese Auswahl zweifellos subjektiv und unvollständig.

\section{Bildgebende Diagnostik}

Neben der klinischen Untersuchung ist die konventionelle Röntgenbilddiagnostik die Standardbildgebung (Ausgangsbild) und bleibt dies vorläufig auch. Auch in der Zeit der vielzeiligen Spiral-CT mit multiplanarer Rekonstruktion erscheint das a.-p. Röntgenbild des Beckens bis auf weiteres unverzichtbar zur Erstbeurtei- lung [13]. Insbesondere, wenn eine umgehende Indikation zur CT gestellt wird, wird die nahe Zukunft zeigen, inwieweit die typischen Standardserien (a.-p. plus Ala- und Obturatoraufnahme bei der Azetabulumfraktur, a.-p. plus Inlet- und Outlet-Aufnahme bei Beckenringverletzungen; Fall 1, $\bullet$ Abb. 1, Fall 2, $\bullet$ Abb. 2) durch die entsprechenden CT-Rekonstruktionen ersetzt werden können (Fall 2, - Abb. 2). Volumenorientierte 3DRekonstruktionen bieten eindrückliches Bildmaterial, können bei geringer Dislokation der Frakturen aber die Frakturerkennung erschweren [22]; zur Bewertung der eigentlichen Pfannenregion ist bei Azetabulumfrakturen das „Herausrechnen“ des Femurkopfs nötig.

Die Standardbilddiagnostik bildet derzeit noch die Grundlage der Frakturklassifikation [13]. Wird das primäre Standard- röntgenbild, das etwa zum Frakturausschluss angefertigt wird, fehlinterpretiert, resultiert oft auch eine therapeutische Fehlentscheidung (Fall 1, - Abb. 1).

Die für die Stabilität des Beckenrings relevanten Strukturen liegen dorsal (Fall 3, - Abb. 3). Wird ein aufnahmetechnisch unzureichendes und somit nicht aussagefähiges Bild als solches akzeptiert, ohne dass die Entscheidung zur weiterführenden Bildgebung (CT) bei entsprechender Anamnese und Klinik fällt, können Verletzungen der Iliosakralgelenke und des Sakrums (zumeist B-, aber auch $\mathrm{C}$-Verletzungen) übersehen werden (Fall 3, - Abb. 3). Dies bedeutet nicht, dass „vorsichtshalber“ zum Ausschluss einer Schädigung der posterioren Beckenringstrukturen grundsätzlich ein CT angefertigt werden sollte, da dies beim jungen $\mathrm{Pa}$ tienten zur hohen Strahlenbelastung der 
Gonaden, bei der rasch zunehmenden Zahl älterer und geriatrischer Patienten zu einer Überdiagnostik ohne therapeutische Konsequenz führt. Gerade bei Letzteren ist es ratsam, im Zweifelsfall einen Mobilisationsversuch zu unternehmen und bei persistierenden Beschwerden unter ausreichender Schmerzmedikation und mangelndem Mobilisationsforschritt ein CT in der 2. Woche nach dem Trauma durchzuführen [13].

Zur Bewertung der Azetabulumfraktur des älteren Patienten ist bei der Häufung der instabilen Brüche mit Beteiligung des vorderen Pfeilers und der 2-Pfeiler-Frakturen die CT in der Frühphase der Entscheidungsfindung „konservativ vs. operativ" hilfreich.

\section{Arterielle Blutung nach Beckenringverletzung}

Hochgradig instabile Beckenringverletzungen (meist C-Verletzungen) gehen häufig mit lebensbedrohlichen massiven Blutungen aus den Venenplexus und/oder den Ästen der A. iliaca interna einher. Die i.v. kontrastmittelverstärkte CT bietet eine verlässliche Möglichkeit, frühzeitig relevante Blutungen zu detektieren (Fall 4 ,

- Abb. 4).

Wenngleich die Wertigkeit dieses Verfahrens insoweit umstritten ist, ob eine Leckage spezifisch für eine lebensbedrohliche Blutung im Beckenbereich ist oder prinzipiell bei jeder signifikanten Beckenringverletzung auftritt [13], bestechen doch die Spezifität (kein Kontrastmittelaustritt im Kontrastmittel-CT: keine relevante Blutung) und der negative prädiktive Wert (konsekutiv keine Angiographie/Embolisation erforderlich) dieses Verfahrens [20]. Eine Angiographie und eventuelle Embolisation stehen im Übrigen nicht an primärer Stelle der Verfahrenswahl bei der signifikanten pelvinen Blutung mit Kreislaufinstabilität, sondern die Anlage einer Beckenzwinge und/oder eines Beckenfixateurs [13]. Reicht dies zur Beherrschung der Blutung durch Kompression und Limitierung des intrapelvinen Raums nicht aus, ist abzuwägen, ob eine extraperitoneale Tamponade („packing") oder eine angiographische Embolisation an dieser Stelle zu bevorzugen sind (• Abb. 4). Das entsprechende No-

Trauma Berufskrankh 2009 - 11[Suppl 1]:97-106 DOI 10.1007/s10039-008-1443-4

(c) Springer Medizin Verlag 2008

T. MittImeier · M. Beck

Verletzungen von Becken und Azetabulum. Fehler, Fallstricke und Lösungswege

\section{Zusammenfassung}

Instabile und/oder dislozierte Beckenringund Azetabulumfrakturen sind selten. Diese prognostisch ernsten Verletzungen betreffen oft mehrfach Verletzte oder Polytraumatisierte. Ihr Risikopotenzial und die Komplikationsraten sind hoch. Entsprechend den meist limitierten Erfahrungen mit diesen Frakturen resultieren zahlreiche Fehlermöglichkeiten in Diagnostik und Therapie, die das Gesamtresultat ungünstig beeinflussen können. Deshalb sollten Betroffene auf Zentren mit entsprechender Erfahrung konzentriert werden. Obwohl evidenzbasierte Empfehlungen weitgehend fehlen, ist es ratsam, auf Expertenkonsens basierenden diagnostischen und therapeutischen Algorithmen zu folgen. Bei der Wahl der an die individuelle Verletzungskonstellation adaptierten Behandlung sollte frühzeitig ein geeigneter Behandlungspfad mit minimierten Risikoaussichten eingeschlagen werden. Eine Auswahl typischer Fehlerkonstellationen der frühen posttraumatischen Phase sowie der jeweiligen Lösungsansätze wird in diesem Beitrag aufgegriffen und soll das Konzept einer entsprechenden Fehlervermeidungsstrategie untermauern. Optionen der Qualitätskontrolle können einen entsprechenden Baustein bilden.

\section{Schlüsselwörter}

Beckenfrakturen - Azetabulumfrakturen .

Management $\cdot$ Komplikationen $\cdot$ Risiken

\section{Injuries of the pelvis and acetabulum. Mistakes, pitfalls, and solutions}

\section{Abstract}

Unstable and dislocated fractures of the pelvis and acetabulum represent rare lesions with a serious prognosis and are often found in multiply injured or polytraumatized patients. The specific risk potential and complication rates are high. A variety of potential errors and pitfalls in diagnosis and therapy can adversely affect the final outcome and correspond to limited individual experience with these fracture entities. Consequently, patients with unstable and/or dislocated pelvic or acetabular fractures should be transferred to institutions in which these fracture patterns are frequently treated. Although evidence-based recommendations are widely lacking, it appears advisable to follow the diagnostic and therapeutic algorithms based on expert opinion and adapt them to the individual fracture pattern to develop a treatment concept with minimized risks. A selection of typical errors of the early posttraumatic phase and their potential solutions is the basis of the present review and underlines a strategy of error prevention. Quality control options may be a useful component.

\section{Keywords \\ Pelvic fractures - Acetabular fractures Management $\cdot$ Complications · Pitfalls}




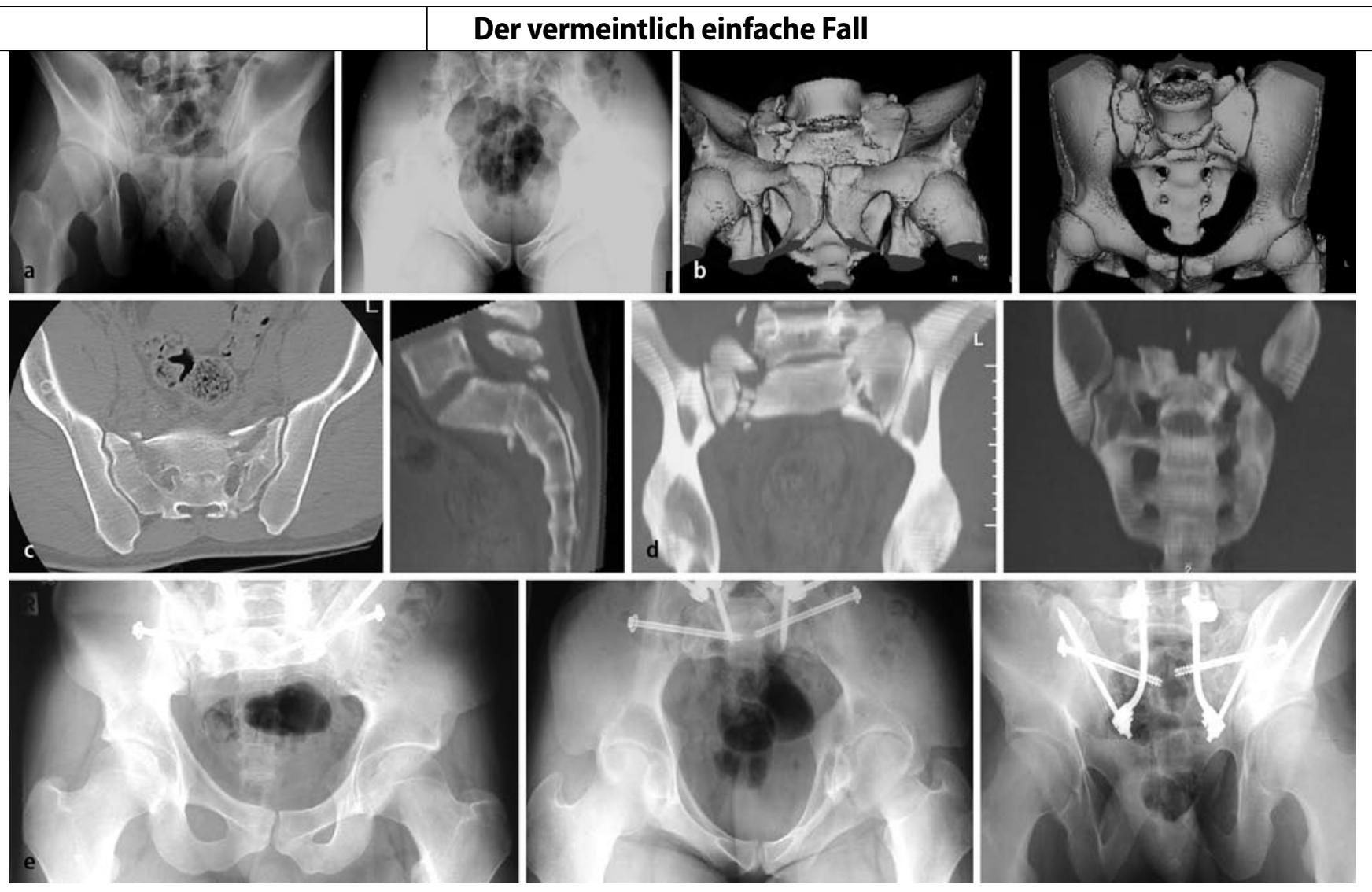

Abb. 2 \ Fall 2, 21-Jähriger mit isolierter bilateraler transforaminaler Sakrumfraktur AO 61C3.3), a Outlet- und Inlet-Aufnahme, b 3D-Oberflächenrekonstruktion in analoger Projektion, c CT-Nativschnitt und sagittale 2D-Rekonstruktion, $\mathbf{d}$ volumenorientierte Rekonstruktion, e a.-p., Inlet- und Outlet-Aufnahme nach Versorgung mit triangulärer bilateraler spinopelviner Transfixation, weitere Erläuterungen s. Kasuistik
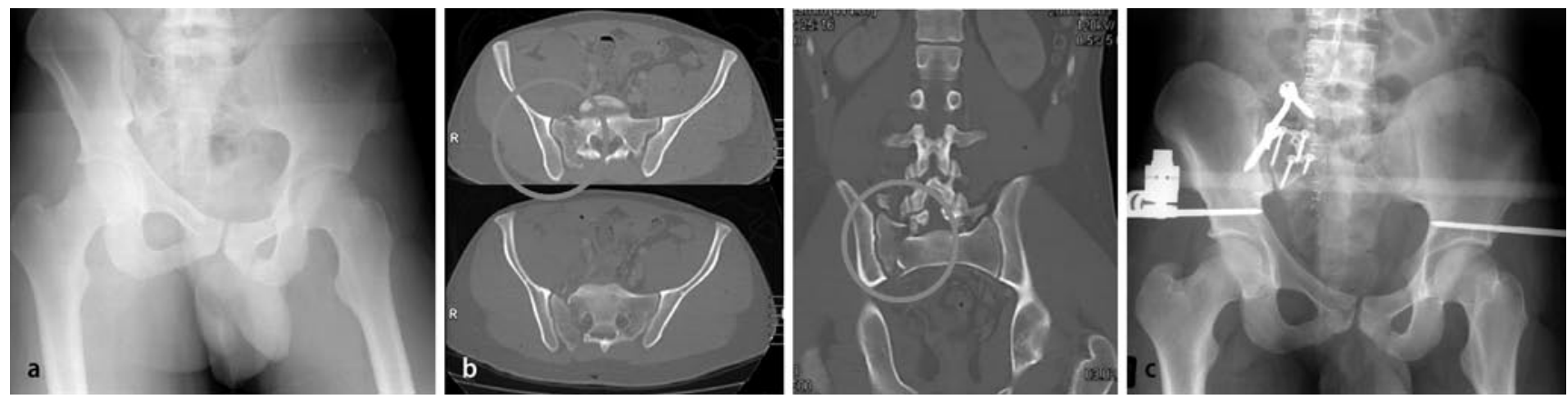

Abb. $3 \Delta$ Fall 3, 24-Jähriger mit isolierter Beckenringverletzung AO 62C1.3, a unzureichende a.-p. Aufnahme des Beckens, b CT mit koronarer Rekonstruktion: C-Verletzung mit vertikalem Versatz, Abriss des Querfortsatzes von LWK5 rechts, mehrfragmentärer Stauchungsfraktur des Sakrums, c offene Reposition, weitere Erläuterungen s. Kasuistik

fallzeitmanagement - sicherlich auch die Frage, ob zum betreffenden Zeitpunkt ein in der interventionellen Angiographie erfahrener Operateur zur Verfügung steht - ist für die Prognose des Patienten entscheidend.

\section{Begleitverletzungen}

Frakturen des Beckenrings und des Azetabulums treten selten isoliert auf. Bei mehr als der Hälfte der Patienten finden sich zusätzliche Brüche [5, 7], der Anteil Polytraumatisierter beträgt bis über $30 \%$ $[2,7]$. Ist die Verletzung der Beckenregion klinisch besonders augenfällig und unterbleibt eine systematische Suche nach weiteren Verletzungen entsprechend dem Polytraumaalgorithmus, kann eine relevante Begleitverletzung primär übersehen werden (- Abb.5). Somit sollte prinzipiell gerade beim Rasanztrauma von „okkulten“ Begleitverletzungen ausgegangen und danach gefahndet werden.

\section{Fehleinschätzung/Non- Compliance bei Mobilisation}

Im Regelfall ist nach Beckenringverletzung eine Teilbelastung bis zur sicheren osteoligamentären Heilung indiziert.

Bei dem in Fall 4 (D Abb. 4, 6) geschilderten Beispiel jedoch kam es durch Sturz aus dem Bett zur Redislokation der Montage und zusätzlich zu einer additiven Sakrumfraktur entlang der Sakrumschrauben, was eine erneute ope- 

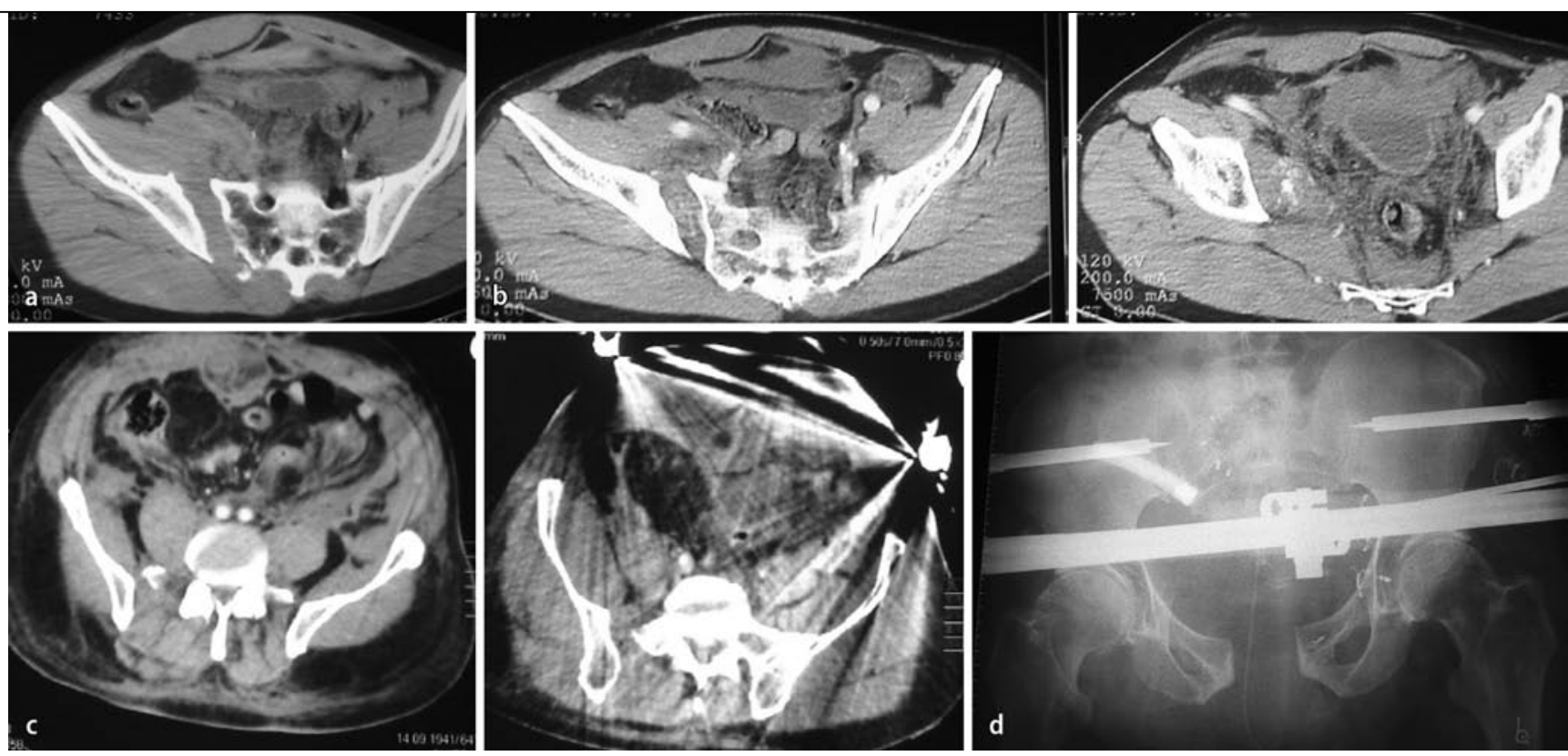

Abb. $4 \Delta$ Fall 4, 62-Jähriger mit AO-Verletzung 61C1.2, a,b Kontrastmittel-CT: Nachweis einer erheblichen aktiven Blutung extraperitoneal, c,d Kontrastmittel-CT: Nachweis der trotz Beckenzwinge und Fixateur-externe-Anlage persistierenden Blutung, Embolisation der aktiv blutenden A.-iliaca-interna-Äste, weitere Erläuterungen s. Kasuistik

rative Versorgung erforderlich machte (s. unten).

\section{Unzureichende Primärstabilität nach operativer Versorgung}

Unbefriedigende Repositionsresultate bei der Azetabulumfraktur und residuale Fehlstellungen bei der Beckenringfraktur führen regelhaft zu schlechteren Resultaten [7, 10, 22]: Matta [10] und Deo et al. $[2,10]$ beobachteten eine 3 -mal höhere Rate einer posttraumatischen Arthrose bei nichtanatomischem (45\%) als bei anatomischem Repositionsergebnis (16\%).

Der für die Reposition geeignete $\mathrm{Zu}$ gang ist sicherlich eine wesentliche Voraussetzung für ein entsprechend gutes Resultat [13]. Lässt sich - trotz korrekter Zugangswahl - etwa bei einer seltenen Kombinationsfraktur der vorderen Azetabulumwand, des vorderen Pfeilers und der quadrilateralen Fläche ( $\mathbf{D}$ Abb. 7a,b) ein grenzwertiges Repositionsergebnis durch unzureichende Stabilität nicht bis zur knöchernen Durchbauung halten, resultiert beim jüngeren Patienten nach Redislokation hieraus zwangsläufig eine Revisionsindikation unter primärem Gelenkerhalt. Problematisch ist dabei die sichere Fixation der quadrilateralen Fläche [11]. Kommt zudem eine posttraumatische Femurkopfnekrose hinzu, ist ein sekundärer Gelen- kersatz unvermeidlich ( $\mathbf{\bullet}$ Abb. 7g), der bei entsprechendem Pfannendefekt meist einer Unterfütterung mit Eigenknochen und Spezialimplantaten bedarf. Nicht zuletzt wegen der schwierigen Ausgangssituation bei der posttraumatischen Arthrose und der vorbestehenden Narbensituation nach rekonstruktivem Voreingriff sind die funktionellen Resultate und die Standzeiten der sekundären Endoprothetik deutlich schlechter als bei der Prothetik bei primärer Koxarthrose $[1,11]$.

Ein primärer Gelenkersatz der Hüfte bei der Azetabulumfraktur des jüngeren Patienten ist lediglich bei simultanen Schenkelhalsfrakturen (Pipkin-IV-Fraktur) oder vorbestehenden Gelenkschäden zu erwägen. Ansonsten ist ein primärer endoprothetischer Gelenkersatz - mit [11] oder ohne Osteosynthese [1], häufig aber mit der Notwendigkeit, spezielle Implantate wie Pfannenabstützschalen einsetzen zu müssen - eine reliable Option für den älteren Patienten [13]. Der ansonsten potenziell anstehende Zweiteingriff wird so vermieden, die Ergebnisse des primären Gelenkersatzes scheinen über denen der sekundär erforderlichen endoprothetischen Versorgung zu liegen $[1,11]$.

\section{Qualitätskontrolle}

Ein valider Beleg der erreichten Behandlungsqualität (z. B. intraoperative Reposition, korrekte Implantatlage) ist nicht allein wegen potenzieller späterer juristischer Implikationen, sondern v. a. zur Sicherung des klinisch-wissenschaftlichen Zusammenhangs zwischen Repositionsresultat und Outcome relevant. Zwar wird die Wertigkeit einer postoperativen CTKontrolle noch kontrovers diskutiert [13], nicht zuletzt weil der bisherige Goldstandard der postoperativen Bildgebung die Standardröntgenuntersuchung darstellt $[4,6]$ und biologische Parameter der jeweiligen Verletzung und Versorgung hiermit nicht detektiert werden können, doch gibt diese Form der postoperativen Bilddokumentation dem Operateur ein gutes Instrument an die Hand, residuale Fehlstellungen nach Rekonstruktion und die adäquate und gesichert extraartikuläre Implantatlage zu dokumentieren, somit „Schwachstellen“ der spezifischen Versorgung nachzuweisen $(\mathbf{A b b} . \mathbf{8 c}, \mathbf{d})$. Hieraus kann neben einem persönlichen Lerneffekt auch eine Indikation für eine operative Frührevision abgeleitet werden. Moderne Formen der intraoperativen Bildgebung (z. B. 3D-Bildverstärker, $\square$ Abb. 8c) sind hierbei von Vorteil, da bei mittlerweile guter Abbildungsqualität und limi- 


\section{Der vermeintlich einfache Fall}
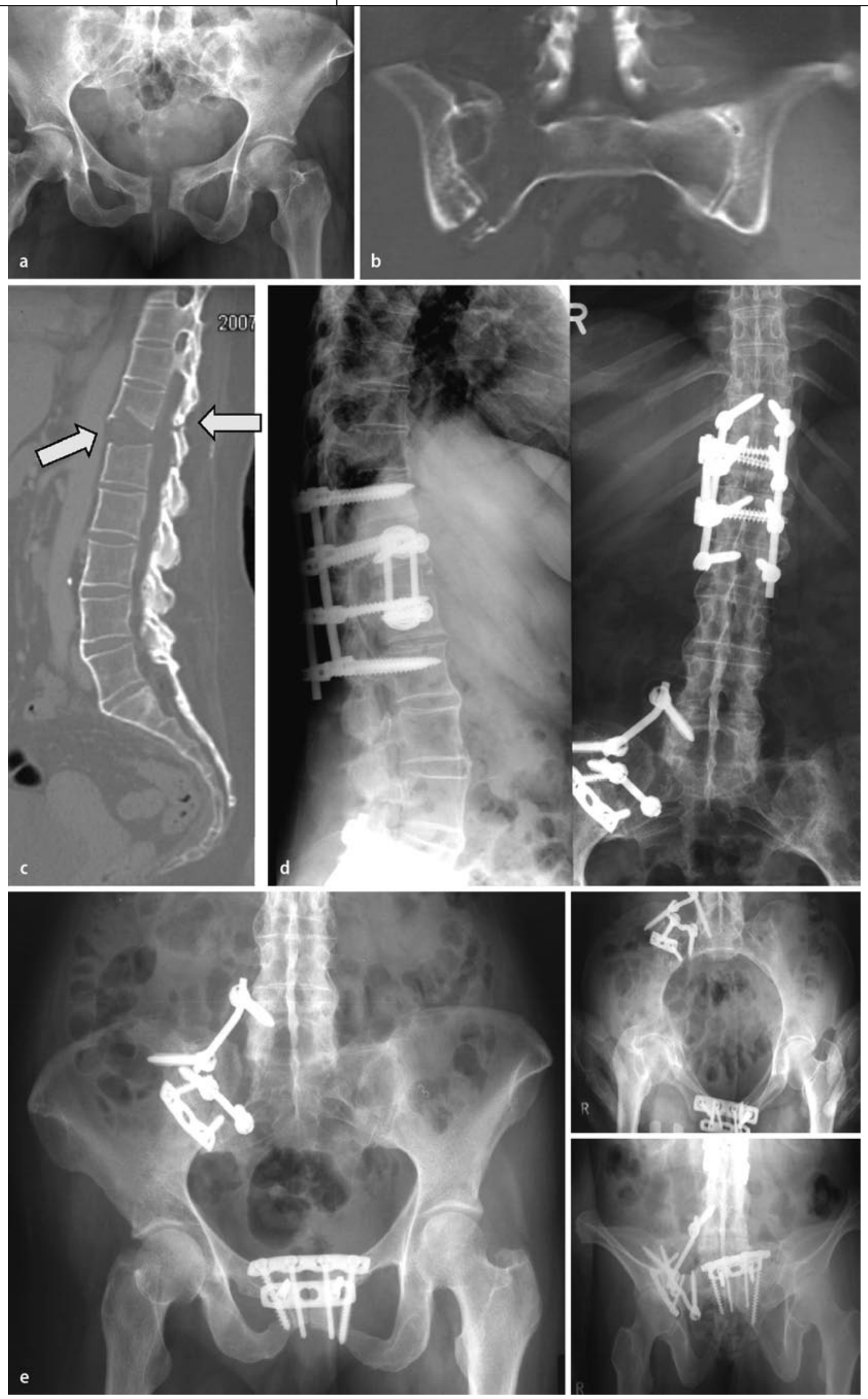

Abb. $5<$ Fall 5, 50-jährige Polytraumapatientin (AO 61C1.2), a,b Symphysensprengung und transiliosakrale Luxationsfraktur rechts, c gleichzeitige BVerletzung der Wirbelsäule BWK12/LWK, d dorsoventrale mehrsegmentale Stabilisation, e ORIF der Beckenringfraktur, weitere Erläuterungen s. Kasuistik 

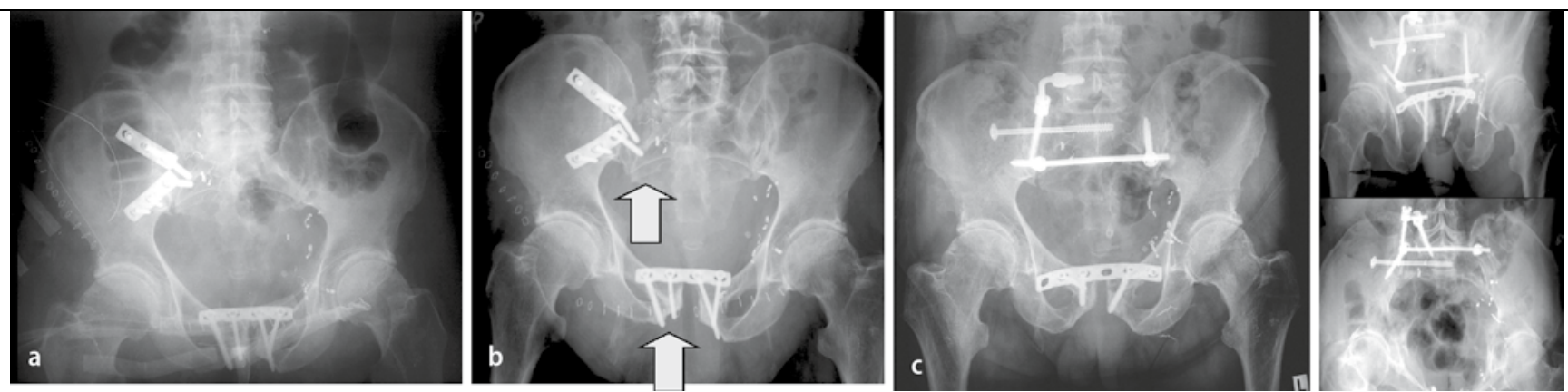

Abb. $6 \Delta$ Fall 4, a postoperatives Versorgungsbild, b Redislokation der Montage, Fraktur des Sakrums periimplantär, c Implantatentfernung, Re-ORIF und erneute Embolisation von A.-iliaca-interna-Ästen, weitere Erläuterungen s. Kasuistik
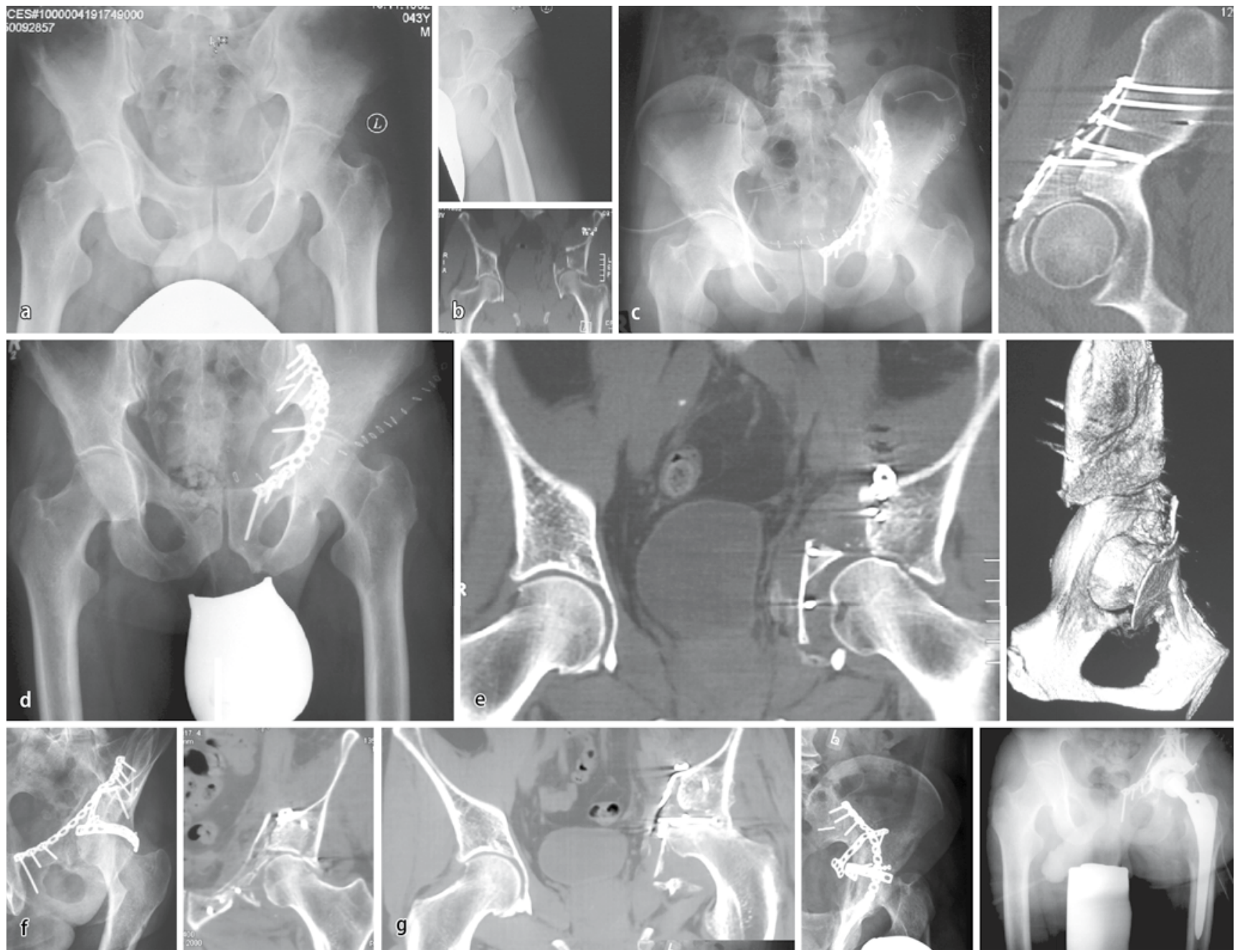

Abb. 7 \ Fall 6, 43-jähriger Patient, a,b AO-Verletzung 62B3.2 mit erheblicher Trümmerzone der vorderen Azetabulumwand, des vorderen Pfeilers, der quadrilateralen Fläche, c inkomplette Reposition (intraartikuläre Stufe, Pfanneninkongruenz), d,e weitere Dislokation unter Mobilisation, $\mathbf{f}$ residuale Inkongruenz der Hüftpfanne nach Revision, $\mathbf{g}$ Teilimplantatentfernung und Hüft-TEP, weitere Erläuterungen s. Kasuistik

tiertem Zeitaufwand dem Operateur die Chance geboten wird, ggf. intraoperativ weitere Nachkorrekturen vorzunehmen, also die postoperative CT-Untersuchung und einen evtl. Zweiteingriff entbehrlich zu machen. Der Vergleich zwischen der intraoperativen $3 \mathrm{D}$-Bildverstärkung und einem postoperativen Standard-CT mit multiplanerer Rekonstruktion scheint dies zu untermauern ( $\mathbf{\square}$ Abb. 8d-f).

Auch sämtliche geringinvasiven Operationsverfahren $[3,17,19,21]$ werden einen entsprechenden bildhaften Beleg benötigen, um eine Gleichwertigkeit hin- sichtlich des erzielbaren Rekonstruktionsergebnisses mit konventionellen $\mathrm{Zu}$ gängen zu untermauern. Der Anwendung der intraoperativen Navigation in der Becken- und Azetabulumchirurgie wird prospektiv im Licht der bisherigen 

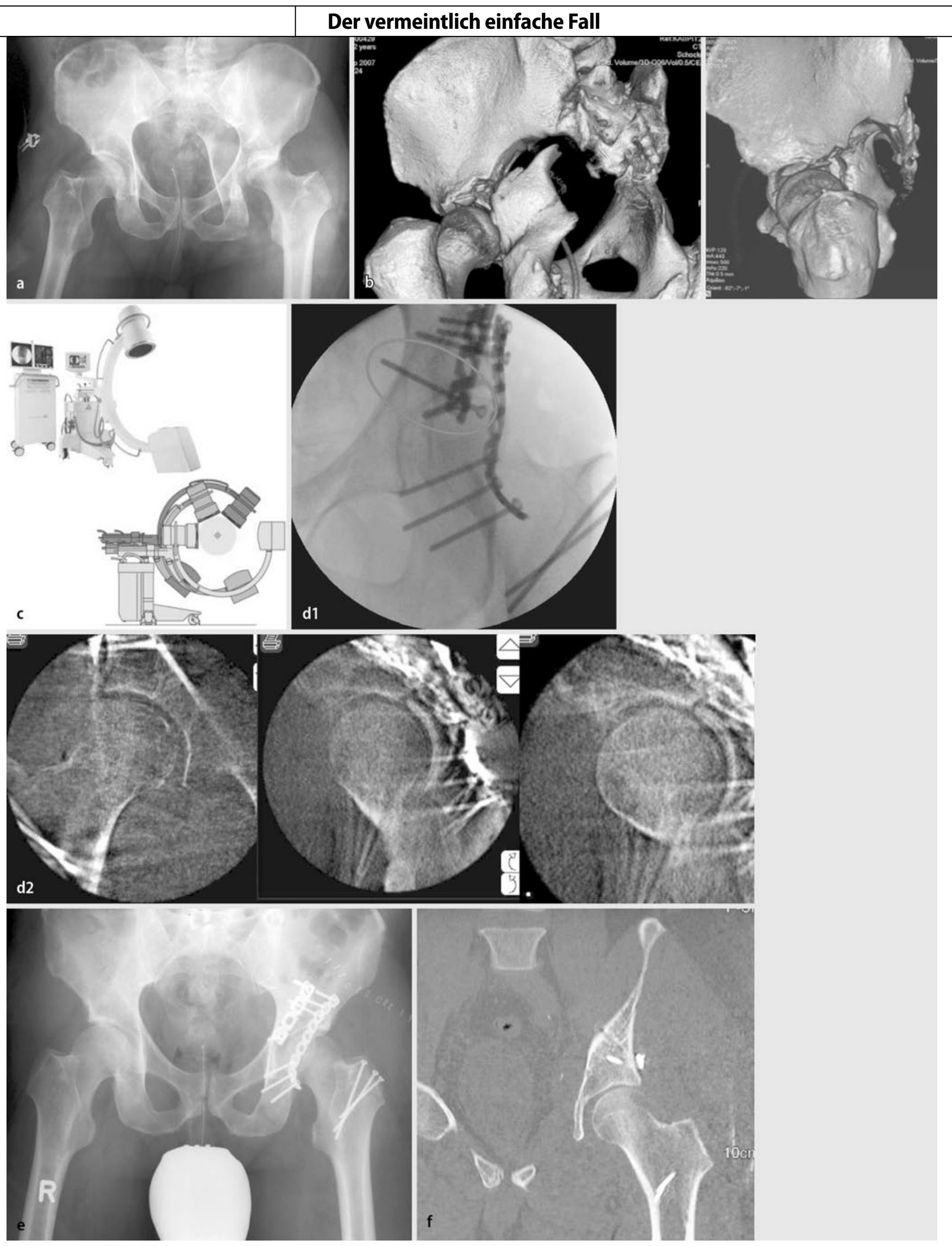

Abb. 8 \ Fall 7, 52-jähriger Polytraumapatient (AO 62A2.3), a,b erhebliche Impressionszonen und osteochondrale Frakturen im Pfannendachbereich, $\mathbf{c}$ intraoperative Kontrolle von Reposition und Implantatlage nach ORIF mit 3D-Bildverstärker, $\mathbf{d}$ Entfernung der markierten Zugschraube und nochmalige 3D-Bildverstärker-Kontrolle, e,f postoperatives a.-p. Röntgenbild und 2D-Rekonstruktion nach CT, weitere Erläuterungen s. Kasuistik 
Resultate vermutlich weitere Bedeutung zukommen $[13,21]$.

\section{Kasuistik}

Fall 1. Die 32-jährige Patientin war im europäischen Ausland gestürzt und nach Vorlage der a.-p. Beckenübersichtsaufnahme (• Abb. 1a) mit "stabiler“ isolierter vorderer Beckenringfraktur unter starken Beschwerden voll mobilisiert worden. Die rechtsseitige Sakrumfraktur wurde erst 10 Tage nach dem Trauma und der Rückkehr in die Heimat diagnostiziert (• Abb. 1b).

Die Fraktur wurde reponiert und mittels supraazetabulärem Fixateur externe und transiliosakraler Stellschraube stabilisiert ( $\bullet$ Abb. 1c). In $\bullet$ Abb. 1d,e sind die Beckenübersichts- und Inlet-Aufnahme nach Entfernung des Fixateurs und Vollmobilisation dargestellt.

Fall 2. Bei dem 21 Jahre alten Mann lag eine isolierte bilaterale transforaminale Sakrumfraktur AO 61C3.3 („blow-out-fracture") vor. Die Diagnose erfolgte mittels Outlet- und Inlet-Aufnahme, 3 D-Oberflächenrekonstruktion in analoger Projektion, CT-Nativschnitt und sagittaler 2D-Rekonstruktion sowie volumenorientierter Rekonstruktion ( $\bullet$ Abb. 2a-d).

Nach Reposition wurde die Verletzung mit triangulärer bilateraler spinopelviner Transfixation versorgt (• Abb. 2e).

Fall 3. Bei dem 24 Jahre alten männlichen Patienten war es bei einem Motorradunfall $\mathrm{zu}$ einer isolierten Beckenringverletzung (AO 62C1.3) gekommen. Die a.-p. Aufnahme des Beckens (• Abb. 3a) war lagerungs- und aufnahmetechnisch unzureichend. Die CT-Aufnahmen mit koronarer Rekonstruktion belegten eine CVerletzung mit vertikalem Versatz sowie Abriss des Querfortsatzes von $\mathrm{LWK}_{5}$ rechts und eine mehrfragmentärer Stauchungsfraktur des Sakrums (• Abb. 3b).

Die Versorgung erfolgte durch offene Reposition der Sakrumfraktur mit winkelstabiler Platte und spinopelviner Transfixation bzw. supraazetabulärem Fixateur externe.

Fall 4. Bei dem 62 Jahre alten männlichen Patient mit einer AO-Fraktur 61C1.2 wur- de mittels Kontrastmittel-CT eine erhebliche aktive Blutung im Extraperitonealraum nachgewiesen, die trotz Beckenzwinge und Anlage eines supraazetabulären Fixateur externe persistierte. Bei der somit erforderlichen operativen Versorgung wurden die aktiv blutenden Äste der A. iliaca interna („,coiling“) embolisiert (• Abb. 4) und eine innere Osteosynthese vorgenommen.

Im Rahmen eines Durchgangssyndroms kam es 3 Tage nach der definitiven Versorgung zum Sturz aus dem Bett und dabei zur Redislokation der Montage sowie einer additiven Fraktur entlang der Sakrumschrauben. Die konsekutive Rekonstruktion beinhaltete eine Sakrum übergreifende Reosteosynthese nach Entfernung der ventralen unilateral das Iliosakralgelenk übergreifenden Implantate, eine Distraktionsspondylodese und eine neuerliche Stabilisierung der Symphysenruptur. Ferner war eine abermalige angiographische Embolisation erforderlich, da sich nach Reinstabilität eine neuerliche arterielle Blutung aus A.-iliaca-interna-Ästen manifestierte ( $\bullet$ Abb. 6).

Fall 5. Bei der 50-jährigen Polytraumapatientin mit bekanntem Morbus Bechterew lagen eine AO-Verletzung 61C1.2 mit Symphysensprengung und transiliosakraler Luxationsfraktur rechts sowie gleichzeitig eine B-Verletzung der Wirbelsäule BWK12/LWK1 ohne neurologisches Defizit vor.

Die Versorgung erfolgte durch dorsoventrale mehrsegmentale Stabilisation sowie ORIF der Beckenringfraktur (• Abb. 5).

Fall 6. Bei dem 43-jährigen Mann (AO 62B3.2) bestand eine erhebliche Trümmerzone der vorderen Azetabulumwand, des vorderen Pfeilers sowie der quadrilateralen Fläche.

Aufgrund des inkompletten Repositionsergebnisses mit intraartikulärer Stufe und Inkongruenz der Pfanne kam es unter Mobilisation zu einer weiteren Dislokation. Nach der Revisionsoperation über einen doppelten Zugang (ilioinguinal und Kocher-Langenbeck-Zugang) lag dennoch eine residuale Inkongruenz der Hüftpfanne vor.
Im weiteren Verlauf entwickelte sich binnen 6 Monaten eine Femurkopfnekrose mit konsekutiver Indikation zur Teilimplantatentfernung und Hüft-TEP (• Abb. 7).

Fall 7. Der 52-jährige männliche Polytraumapatient (AO 62A2.3) wies erhebliche Impressionszonen und osteochondrale Frakturen im Pfannendachbereich auf.

Nach ORIF über einen erweiterten dorsalen Zugang mit Trochanter-FlipOsteotomie wurden die Reposition und die Implantatlage intraoperativ mit dem 3 D-Bildverstärker kontrolliert. Die dabei identifizierte „Schwachstelle“ der operativen Versorgung wurde in gleicher Sitzung behoben und das Ergebnis erneut intraoperativ kontrolliert.

Die postoperative Röntgenuntersuchung und CT-Rekonstruktion bestätigten den Befund der intraoperativen Kontrollaufnahme ( $\bullet$ Abb. 8).

\section{Diskussion und Schlussfolgerung}

Dislozierte Beckenring- und Azetabulumfrakturen sind zumeist keine einfachen Verletzungen, selbst wenn dies die Namensgebung der Klassifikation bisweilen suggerieren könnte.

Ein standardisierter diagnostischer und therapeutischer Algorithmus erscheint heute wesentlich dafür, das Ausmaß der individuellen Verletzung korrekt analysieren und zeitgerecht adäquate therapeutische Schritte einleiten zu können $[11,13,22]$. Die relative Seltenheit dieser Verletzungen lässt es sinnvoll erscheinen, Betroffene auf geeignete Zentren zu konzentrieren, die über das gesamte Diagnostik- und Behandlungsarmamentarium einschließlich des Komplikationsmanagements verfügen.

Komplextraumen des Beckens sind lebensbedrohliche Verletzungen mit einer hohen Mortalität $[8,14]$. Eine Fehlervermeidungsstrategie ist ein wesentliches Element der individuellen Entscheidungsfindung und präoperativen Planung, da die Zahl der Komplikationen auch in der aktuellen Literatur als hoch zu bezeichnen ist $[2,5,9,18,22]$. Somit gilt es, im Rahmen der Versorgungsplanung den geeigneten Zugang unter Beachtung der Prin- 
zipien der Weichteilschonung und die adäquate Repositions- und Stabilisationstechnik auszuwählen, wobei in der Azetabulumchirurgie die Mehrzahl der Situationen mit der Anwendung eines der 3 Standardzugänge (Kocher-Langenbeck-, ilioinguinaler sowie erweiterter iliofemoraler Zugang, ggf. auch in Kombination) beherrscht werden kann $[4,6,10,13]$.

Eine adäquate Reposition mit residualen Dislokationen $<5 \mathrm{~mm}$ bei der Rekonstruktion des hinteren Beckenrings und die stabile Wiederherstellung der Kongruenz der Hüftpfanne mit möglichst stufenfreier Rekonstruktion der Domregion bei der Azetabulumfraktur sind prognoserelevante Parameter [5, 10, 13]. Allerdings lässt sich die Prognose der Verletzung in Kenntnis des Einflusses weiterer Kofaktoren nicht allein auf die anatomische Rekonstruktion verkürzen [22]. Letztere ist aber immerhin vom Operateur beeinflussbar und somit kommt der intra- und postoperativen Dokumentation der erzielten Reposition und der korrekten Implantatlage eine wesentliche Bedeutung zu (Qualitätskontrolle). Verfahren der intraoperativen $3 \mathrm{D}$-Bildgebung und der kontrollierten Implantatpositionierung, etwa mit Hilfe der Navigation, können den Operateur hierbei unterstützen und senken die „Hemmschwelle“ für eine allfällige Korrektur, da im Rahmen ein- und desselben Eingriffs ggf. korrigiert werden kann [21]. Ein derartiges Vorgehen unterstützt die Anwendung minimalinvasiver Verfahren, für die allerdings die gleichen Anforderungskriterien hinsichtlich der Güte der Reposition gelten wie für ein offen rekonstruktives Vorgehen [17].

Die Zunahme geriatrischer Patienten mit instabilen Beckenring- und Azetabulumfrakturen schafft spezifische Herausforderungen hinsichtlich der Implantatverankerung im osteoporotischen Knochen bzw. des Einsatzes eines alleinigen primären Gelenkersatzes oder eines Kombinationsverfahrens aus Ostesynthese und Gelenkersatz $[1,11,13]$. Da nicht zuletzt bei derartigen Entitäten heute noch zahlreiche Problemfelder offene Fragen hinterlassen, bietet die Option eines europaweiten Expertennetzwerks und Registers nach dem Muster der AG Becken der DGU die Chance einer validen Datenba- sis für entsprechende Antworten auch im Sinne einer weiteren Minimierung der Komplikationsrate [15].

\section{Korrespondenzadresse}

Prof. Dr. T. Mittlmeier

Abteilung für Unfall- und Wiederherstellungschirurgie, Chirurgische Klinik und Poliklinik, Universität Rostock,

Schillingallee 34, 18055 Rostock

thomas.mittlmeier@med.uni-rostock.de

Interessenkonflikt. Der korrespondierende Autor gibt an, dass kein Interessenkonflikt besteht.

\section{Literatur}

1. Cochu G, Mabit C, Gougam T et al. (2007) L'arthroplastie totale de hanche dans le traitement des fractures récentes de l'acétabulum du sujet âgé. Rev Chir Orthop Reparatrice Appar Mot 93: 818-827

2. Deo SD, Tavares SP, Pandey RK et al. (2001) Operative management of acetabular fractures in $\mathrm{Ox}$ ford. Injury 32: 581-586

3. Jakob M, Droeser R, Zobrist R et al. (2006) A less invasive anterior intrapelvic approach for the treatment of acetabular fractures and pelvic ring injuries. J Trauma 60: 1364-1370

4. Judet R, Judet J, Letournel E (1964) Fractures of the acetabulum: classification and surgical approaches for open reduction. Preliminary report. J Bone Joint Surg Am 46: 1615-1646

5. Kumar A, Shah NA, Kershaw SA et al. (2005) Operative management of acetabular fractures. A review of 73 fractures. Injury $36: 605-612$

6. Letournel E, Judet R (1993) Fractures of the acetabulum, 2nd edn. Springer, Berlin Heidelberg New York

7. Leutenegger A, Planta AR von, Ruedi T (1999) Epidemiologie und Nachuntersuchungsergebnisse operativ behandelter Beckenfrakturen. Swiss Surg 5: 47-54

8. Lunsjo K, Tadros A, Hauggaard A et al. (2007) Associated injuries and not fracture instability predict mortality in pelvic fractures: a prospective study of 100 patients. J Trauma 62: 687-691

9. Mason WT, Khan SN, James CL et al. (2005) Complications of temporary and definitive external fixation of pelvic ring injuries. Injury 36: 599-604

10. Matta JM (1996) Fractures of the acetabulum: accuracy of reduction and clinical results of fractures operated within three weeks after the injury. J Bone Joint Surg Am 78: 1632-1645

11. Mears DC (1999) Surgical treatment of acetabular fractures in elderly patients with osteoporotic bone. J Am Acad Orthop Surg 7: 128-141

12. Plaisier BR, Meldon SW, Super D et al. (2000) Improved outcome after early fixation of acetabular fractures. Injury 31: 81-84

13. Pohlemann T, Culemann U (2007) Summary of controversial debates during the $5^{\text {th }}$ "Homburg Pelvic Course" 13-15 September 2006. Injury 38: 424-430

14. Pohlemann T, Tscherne $H$, Baumgaertel F et al. (1996) Beckenverletzungen: Epidemiologie, Therapie und Langzeitverlauf. Übersicht über die multizentrische Studie der Arbeitsgruppe Becken. Unfallchirurg 99: 160-167
15. Pohlemann T, Tosounidis G, Bircher M et al. (2007) The German multicentre pelvis registry: a template for a european expert network? Injury 38: 416-423

16. Roetman B, Seybold D, Keil D et al. (2006) Langzeitergebnisse nach Azetabulumfrakturen unter Berücksichtigung von heterotopen Ossifikationen. Zentralbl Chir 131: 188-193

17. Rommens PM (2007) Is there a role for percutaneous pelvic and acetabular reconstruction? Injury 38: 463-477

18. Russell GV, Nork SE, Routt CML Jr (2001) Perioperative complications associated with operative treatment of acetabular fractures. J Trauma 51: 10981103

19. Starr AJ, Jones AL, Reinert CM et al. (2001) Preliminary results and complications following limited open reduction and percutaneous screw fixation of displaced fractures of the acetabulum. Injury 32: S-A45-S-A50

20. Stephen DJ, Kreder HJ, Day AC et al. (1999) Early detection of arterial bleeding in acute pelvic trauma. J Trauma 47: 638-642

21. Stöckle U, Schaser K, König B (2007) Image guidance in pelvic and acetabular surgery - expectations, success and limitations. Injury 38: 450462

22. Tornetta P III (2001) Displaced acetabular fractures: indications for operative and nonoperative management. J Am Acad Orthop Surg 9: 18-28

23. Weber K, Kohler H, Vock B et al. (2002) Lebensqualität nach Acetabulum- und Beckenringfrakturen. Orthopade 31: 582-586

24. Wey J, DiPasquale D, Levitt L et al. (1999) Operative treatment of acetabular fractures through the extensile Henry approach. J Trauma 46: 255-260 